\title{
To link or not to link? Empirical guidance for the design of nonlinear text systems
}

\author{
SUSAN H. GRAY \\ New York Institute of Technology, New York, New York \\ and \\ DENNIS SHASHA \\ New York University, New York, New York
}

\begin{abstract}
We compared the effectiveness of two versions of the NetBook data model for nonlinear textone with links and one without. Users who accessed information through specifications based on properties and values alone performed better than users who employed such specifications as well as hypertext links. Protocol analysis revealed that hypertext links were useful when a subject's specifications were unspecific but correct. However, hypertext could rarely compensate for inaccurate specifications. Design recommendations are included.
\end{abstract}

In this study, we use controlled experimentation and protocol analysis for two purposes: (1) to compare linked and nonlinked versions of the NetBook data model in order to contribute to the current debate over user's categorization errors, searching via links, and getting lost in nonlinear systems, and (2) to evaluate the effectiveness of the NetBook knowledge exploration system (Shasha, 1985, 1986).

The NetBook model is based on relational databases augmented with hierarchies on domains and hypertext. The user of information accesses sets of text fragments through menu selection of properties and values and then explores other fragments through hypertext links. The system attempts to linearize the set of fragments chosen by a query in a rational way (e.g., prerequisite information first).

\section{Data Organization, User Interface, and User Errors}

We were concerned with two related aspects of information systems, both of which influence effectiveness: how the data are organized in the database and how easy the interface is to use.

Canas, Safayeni, and Conrath (1985) studied the organization of material in a filing system and found a retrieval success rate of over $90 \%$. They suggest that if we can understand how people classify and search for information in such manual systems of information storage, we should be able to design better automated informa-

The NetBook system was implemented by Leah Beckman, Jose PerezCarballo, and Dana Birkby. We wish to thank them for all their help. The work of Dennis Shasha was partially supported by the National Science Foundation, under Grant DCR8501611, and by the Office of Naval Research, under Grant N00014-85-K-0046. Correspondence may be addressed to Susan H. Gray, Department of Behavioral Sciences, New York Institute of Technology, 1855 Broadway, New York, NY 10023. tion systems. Studies of electronic retrieval have generally shown a lower rate of retrieval success (Frankhuizen \& Vrins, 1980).

These retrieval rates may be lower because unskilled users opt for only the basic searching features of an automated system. Weyer (1982) examined the effect of the interface on users' searching strategies. He employed a dynamic book, Dynabook, with a high-school worldhistory text as the database. He compared it with two other versions: paper, and electronic but nondynamic. In the dynamic book, instead of accessing with a one-level subject index and page numbers as in the nondynamic, electronic book, students accessed the information through an expanded three-level outline that included chapters, sections, and subsections, along with mechanisms for matching patterns. Weyer found that the students learned the elementary features of the system quickly, but that the more elaborate features were seldom used. Although the students preferred the two electronic forms of the book, the nondynamic electronic book was faster to use. The correctness of answers did not vary between conditions.

Many accessible databases for novice users are menu driven, as is NetBook. Otherwise, the user may experience what Landauer, Dumais, Gomez, and Furnas (1982) call "novice freakout." Indeed, novice freakout may even occur with a menu-driven system if the menu is not clear to the user, or if the data in the database are poorly arranged from the user's point of view. As Walker (1981) points out, it is essential that the user have a reasonable knowledge of how the information in the database is organized. Even with that knowledge, however, if the organization of the database is different from what the user expects, there may still be problems.

Landauer et al. (1982) point out that efficient system processing generally takes priority over other considerations in the arrangement of data in a database. The logi- 
cal connections in the users's mind may not match the connections necessary for an efficient information system, because people seldom organize information in a deeply nested hierarchy. Also, people's systems of categorization differ. Studies of videotext systems support the contentions of Dumais and Landauer (1984). In an experimental study of the Prestel Viewdata service, a database containing information about a range of topics (news, financial data, and other reference materials) Frankhuizen and Vrins (1980) asked subjects to find answers to various questions, such as "What language is spoken in Ethiopia?" Almost half of the questions were not answered correctly. In addition, for almost half of the questions, the subjects did not find the answers by using their initial strategy, but rather started over again from the beginning one or more times. In terms of the precision of retrieval, even the successful locations of answers were not efficient. On the average, twice as many pages were retrieved as was necessary. Frankhuizen and Vrins attribute these problems to category overlap and to ambiguities in category naming. One videotext system, for example, would decompose "nightlife" in its hierarchies into supposedly disjoint categories including "attractions" and "entertainment." The problems associated with categorization in a single hierarchy suggest that a knowledge exploration system should support multiple overlapping hierarchies, a decision taken in NetBook.

An additional categorization problem that any system will have is suggested by Walker (1981). In an information system, new developments in a body of knowledge may necessitate the addition of new entries to the index listing, but it can be difficult to reclassify the older text to reflect the changes. The example given by Dumais and Landauer is that if you want to locate computer science books, they are classified under the category "generalities" in the Dewey Decimal System.

\section{Information Links}

One proposal for overcoming categorization problems and false starts has been to use links between information fragments. In his classic article on the future of information systems, Bush (1945) suggested that because the index categories by which information can be stored in a system and the mental processes by which a human classifies information are very different, selection by indexing is not optimal. Rather, since the human mind operates in an essentially nonlinear way by means of association, selection by association should be a better strategy, if it can be mechanized. Bush called this process of linking many items of information "building a trail," ideally with the capacity to branch onto side trails of subtopics, through the available texts.

These ideas are consistent with theories in modern psychology. Take, for example, the theory of "spreading activation." The basic idea here is that each person has a mental dictionary of words linked to other words by means of associated meanings, associated sounds, or associated grammars, forming a sort of spiderweb of points. Speak- ing activates points on this web; each word selected activates an alternate path through it (Motley, 1987).

Yankelovich and Meyrowitz (1985) argue that computers, compared with books, are particularly suited for helping users create connectivity (i.e., webs of related information). In a book, links are usually created only through footnotes or through notes that the user writes in the margins. With electronic media, the organization of materials can be more flexible.

Links between fragments of information may also have drawbacks. Yankelovich and Meyrowitz believe that a disadvantage of electronic information systems is that such systems do not inform users where they are in a document. To follow a trail of links may exacerbate this problem (Negroponte, 1979; Yankelovich \& Meyrowitz, 1985). This contrasts with books, in which the reader can tell if he/she is halfway through or someplace else. Because an electronic book is nonlinear, the middle for one user might be the beginning for another. Therefore, someone following link after link may feel very disoriented. For example, in the FRESS hypertext document system at Brown University, users often found it difficult to remember where they were in the text. Nevertheless, virtually all researchers feel strongly that any electronic document system should include tools for connectivity.

Several nonlinear text systems that utilize links have been described, with Conklin (1987) having provided a recent overview; the largest is currently ZOG/KMS, with 50,000 frames (Akscyn \& McCracken, 1984; Akscyn, McCracken, \& Yoder, 1988; Engelbart, 1984; Fogg, 1984; Herot, 1980; Lowe, 1985; Nelson, 1984; Shasha, 1985, 1986; Shneiderman, 1987; Trigg \& Weiser, 1986). Many different design features have been employed. In Fogg's Living in a Database System, for example, users from inside an entity-relationship tuple can answer queries by examining the database from that tuple's perspectives. Fogg bases his interface on the ideas of Cattell (1980), who describes an interface in which each frame contains a list of adjacent nodes for the user to explore. Herot's (1980) spatial data management system (SDMS) presents information graphically.

Shneiderman (1987) describes a nonlinear system that works through the use of embedded menus: The Interactive Encyclopedia System (TIES, or HyperTIES). Its users can select topics by means of words highlighted in the text and immediately view an article on the selected topic. This contrasts with the more traditional separation of text reading from menu-category selection. When embedded menus are compared with more traditional menus, subjects complete more questions more quickly and also jump more frequently among articles.

\section{EXPERIMENT}

A database consisting of text fragments was created, using the authoring and editing facilities of NetBook (Shasha, 1985, 1986). The text fragments were taken largely from two chapters of an introductory sociology 
text. Some additions and modifications were made where it was thought that information needed to be updated or elaborated upon.

The fragments were broken down by 10 relevant properties and appropriate values (properties correspond to slots in frame-based systems or attributes in database systems). Subvalues, or hierarchies within property domains, were not employed, because we wanted to use only the simplest mechanisms in NetBook. The properties and values were chosen so that one could obtain the same information through more than one query. They were also chosen to be orthogonal as much as possible (i.e., choosing a value of one property should not restrict the possible values of other properties).

The links among the fragments included example, critique, counterargument, compared with, and continuation (linking a fragment to the following one in the original text). Continuation links were also used by NetBook as input to a presentation algorithm that presented adjacent fragments with the properties and values queried in linear order. The continuation order was not violated, unless it was necessary to avoid violating an example link or one of the other types of links. The notion of trying to present the fragments returned by a query in the same order as that in which they appear in the original text may strike nonlinear text adherents as blasphemy, but that heuristic seemed reasonable to us, all else being equal.

The subjects were undergraduate volunteers from The New York Institute of Technology. Fifty were randomly assigned to one of two groups and run individually. The subjects in both groups were permitted to use properties and values to specify text fragments. The difference came about in the use of hypertext links. Participants in the links group were told about the links option and encouraged to use it. Participants in the no-links group were told that the links option on their screen menu could not be used.

An additional 10 subjects were randomly assigned to a third group, for the purpose of making baseline comparisons of speed and accuracy. This group used a paper copy of the text and its table of contents to search for information (paper text group). These subjects were also run individually. The groups had no significant differences in mean grade-point average, and they had been equally exposed to sociology.

Each subject from Group 1 and Group 2 was exposed to a 20-min group introduction to the database and the query system, followed by an individual review and demonstration. In the individual review, the subjects were given a sheet listing the properties and values that organized the text fragments. The sheet also contained a brief outline of how to use the menu system to form queries. The subjects were given $5 \mathrm{~min}$ to review the sheet, and they were told that they could also keep it in front of them and refer to it as the session proceeded. They were also encouraged to ask questions about the procedure at this time. After each subject in Group 1 and Group 2 (the electronic groups) had reviewed the data- base organization and query-procedure outline, the subject underwent a hands-on demonstration.

The subjects were then told to locate, as fast as they could, the information requested in the five questions given them. The presentation order of the questions was rotated in order to measure practice effects. Each search was timed from the point at which the question was presented to the point at which the subject began to record the answer on the answer sheet. In addition, protocols of each subject's menu choices throughout each query were collected (see Figures 1 and 2 for a sample protocol sheet and NetBook's menu choices). If the subject was unable

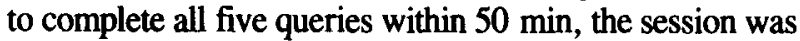
terminated at that point.

\section{RESULTS}

\section{Use of Links}

The links-group subjects were not all equally inclined to use them. Table 1 shows the number of subjects who used the hypertext capabilities of the system, broken down by question. Some questions apparently encouraged the use of links more than others. Questions 3 and 4 were fairly accessible without the use of links, so most subjects searched for an answer through properties and

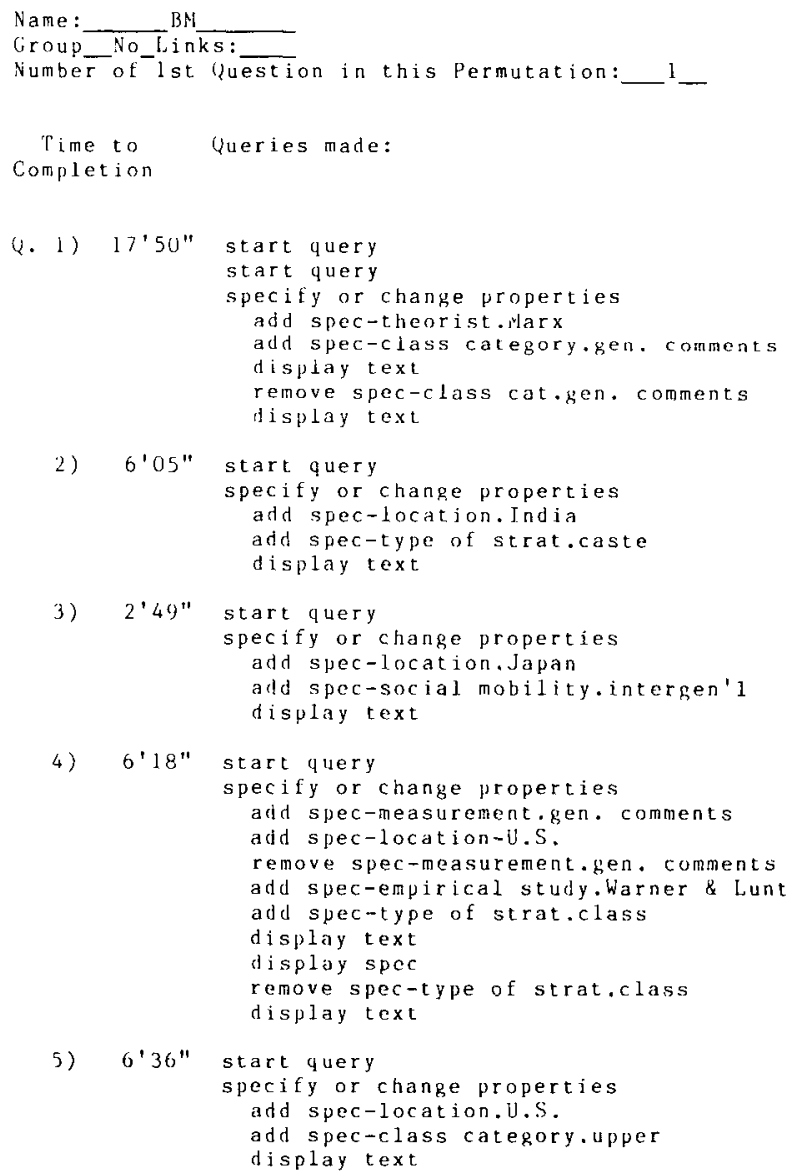

Figure 1. Sample protocol sheet. 


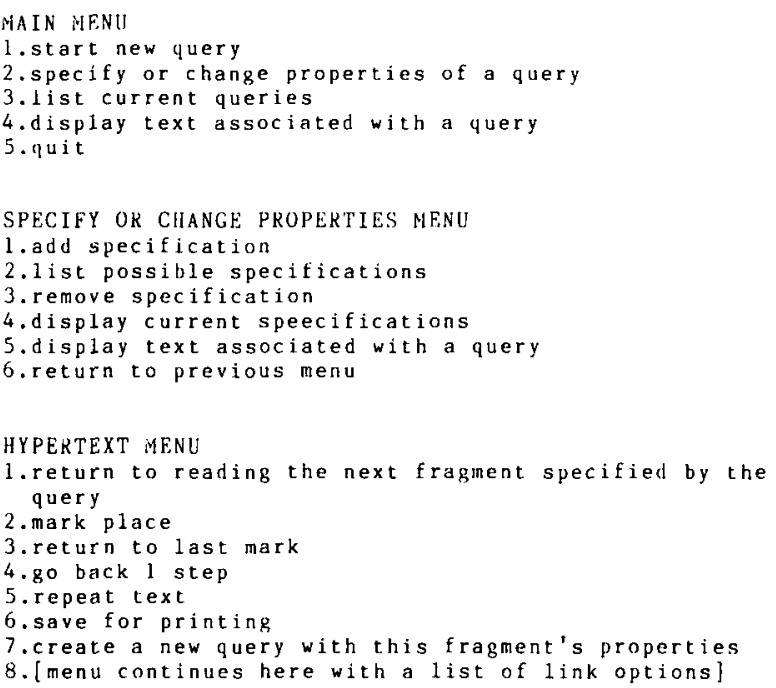

Figure 2. NetBook user menus (version without editing facilities).

values. Informal questioning of the subjects who did not use links, or who used them very sparingly, revealed that some subjects felt overwhelmed by the hypertext menu, despite a review of the menu choices as part of the initial introduction of the subjects to the system.

Table 2 shows the frequencies of hypertext use in queries in the links group. Three subjects would not use the hypertext system at all. Other subjects used it for between one and three queries. The subjects who did not use links, even though links were available, were grouped with those in the no-links group for analyses where applicable.

NetBook's links were sufficient for one to locate information fairly directly with an inadequately specified or incompletely specified query. For example, in the following protocol excerpt, for Question 5 ("The percent of the national wealth that is owned by the richest fifth of Americans.' "), the subject has specified only that she wishes to see all text related to wealth:

$$
\begin{aligned}
& \text { start query } \\
& \text { specify or change properties } \\
& \text { add spec.-form of class inequality.wealth } \\
& \text { display text - hypertext - compared with }
\end{aligned}
$$

Although it took the subject $788 \mathrm{sec}$ to complete the query, which was somewhat longer than the average for the subjects in the no-links group, she needed to follow only one link to obtain the answer. Most of the time the subject spent completing the query was spent hesitating about what to do, rather than doing anything with the system that took a substantial amount of time.

On the other hand, a subject with a query that was not fully specified, or inappropriate, could also become totally lost in the hypertext feature of NetBook. In the following protocol excerpt, the subject spent the entire $50 \mathrm{~min}$ attempting to locate the answer to Question 1 ("'The name of Marx's book, in which he begins to present his ideas on social class."). She never completed the query, but rather went back and forth over the same text links:

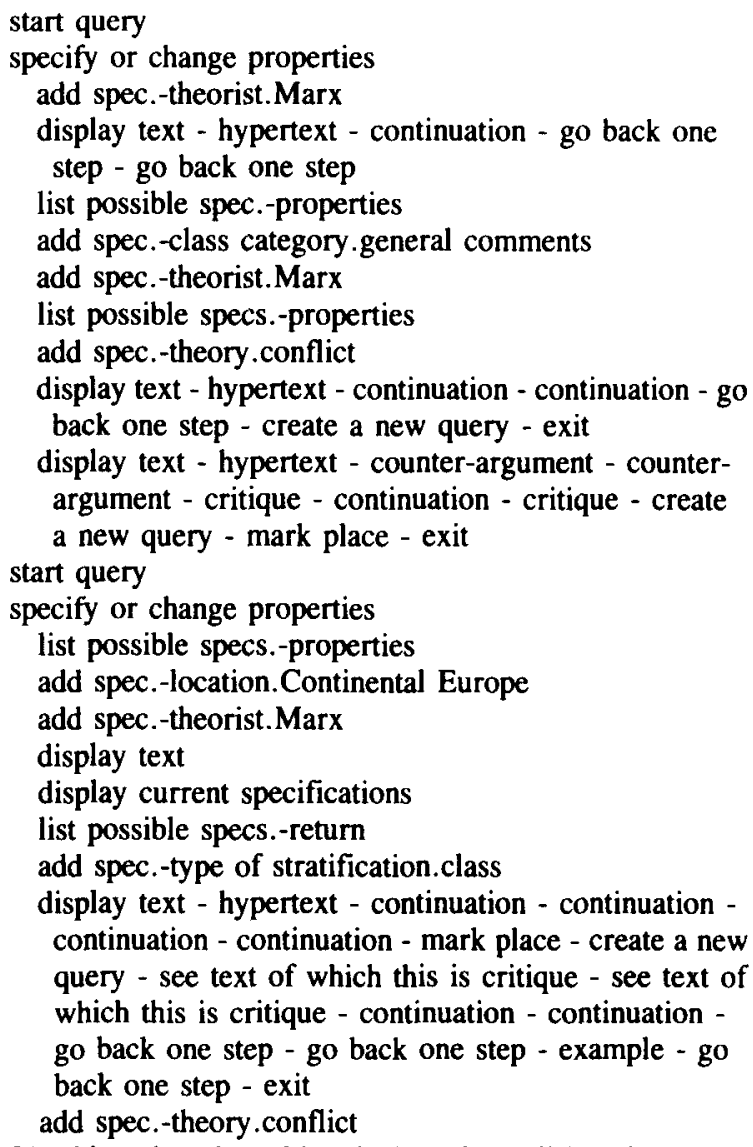

[At this point, the subject had used up all her time.]

The next protocol excerpt illustrates the way a subject may use links to locate information with a poorly specified query. Completion of this query for Question 4 took $737 \mathrm{sec}$. Instead of using the property "empirical study" with the value "Warner and Lunt" and the property

Table 1 Use of Links by Question Number

\begin{tabular}{ccc}
\hline Question & Used Hypertext & $\begin{array}{c}\text { Did Not } \\
\text { Use Hypertext }\end{array}$ \\
\hline 1 & 15 & 3 \\
2 & 10 & 6 \\
3 & 3 & 13 \\
4 & 5 & 15 \\
5 & 10 & 9 \\
\hline
\end{tabular}

Table 2 Frequency of Hypertext Use

\begin{tabular}{cc}
\hline $\begin{array}{c}\text { Number of Queries } \\
\text { Using Hypertext }\end{array}$ & Frequency \\
\hline 0 & 3 \\
1 & 7 \\
2 & 9 \\
3 & 6 \\
\hline
\end{tabular}

Note-Average number of completed queries $=3.28$. Total frequency $=25$. 
"class category" with the value "general comments" in the query, the subject chose to approach her task by guessing at a correct measurement method. She begins by choosing an incorrect method, removes it, chooses another incorrect specification, removes it, adds one that provides only general comments and from there follows links until finding the correct answer:

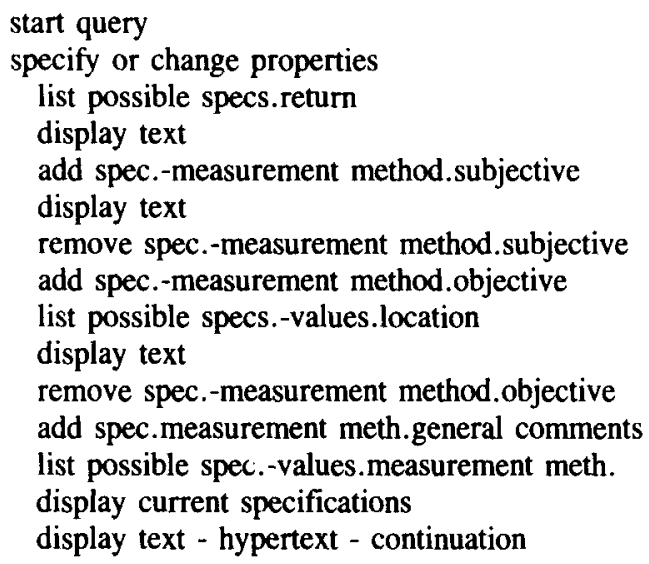

This can be compared with the following protocol excerpt for Question 1, in which the subject uses links not to locate the correct answer, but to realize that he has specified his query incorrectly. He then returns to the menu to adjust his specification and finds the answer. This query took $533 \mathrm{sec}$ to complete:

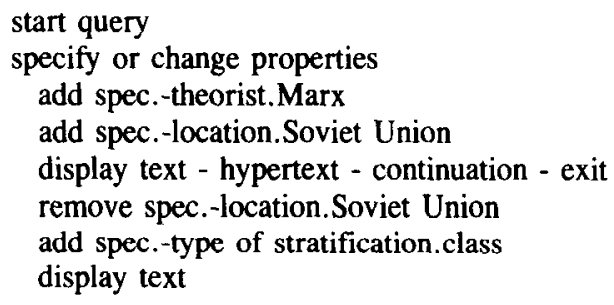

\section{Number of Links}

Table 3 shows the average number of links used for each question by those subjects in the links group who used the system's hypertext capabilities. The maximum

Table 3

Average Number of Links Used by Question Number

\begin{tabular}{ccc}
\hline Question & $M$ & $S D$ \\
\hline 1 & 3.933 & 7.235 \\
2 & 4.500 & 5.276 \\
3 & 3.000 & 3.464 \\
4 & 4.800 & 4.324 \\
5 & 3.100 & 3.143 \\
\hline
\end{tabular}

Table 4

Average Number of Queries Completed by Group

\begin{tabular}{lcc}
\hline Group & $M$ & $S D$ \\
\hline Text & 5.00 & \\
No-links & 4.25 & 1.29 \\
Links & 3.23 & 1.48 \\
\hline
\end{tabular}

Note- $F(2,57)=7.784, p=.0010$.
Table 5

Average Seconds to Complete Each Query

\begin{tabular}{|c|c|c|c|}
\hline Question & Group & $M$ & $S D$ \\
\hline \multirow[t]{2}{*}{1} & $\begin{array}{l}\text { Text } \\
\text { No-links } \\
\text { Links }\end{array}$ & $\begin{array}{r}508.80 \\
570.55 \\
1125.56\end{array}$ & $\begin{array}{l}493.56 \\
526.05 \\
837.28\end{array}$ \\
\hline & \multicolumn{3}{|c|}{$F(2,45)=4.306, p=.0194$} \\
\hline 2 & $\begin{array}{l}\text { Text } \\
\text { No-links } \\
\text { Links }\end{array}$ & $\begin{array}{r}47.70 \\
397.92 \\
877.14\end{array}$ & $\begin{array}{r}15.10 \\
289.37 \\
512.36\end{array}$ \\
\hline \multicolumn{4}{|c|}{$F(2,45)=17.810, p=.0000$} \\
\hline 3 & $\begin{array}{l}\text { Text } \\
\text { No-links } \\
\text { Links }\end{array}$ & $\begin{array}{l}746.00 \\
308.08 \\
493.00\end{array}$ & $\begin{array}{l}434.39 \\
171.28 \\
560.15\end{array}$ \\
\hline \multicolumn{4}{|c|}{$F(2,47)=5.141, p=.0096$} \\
\hline 4 & $\begin{array}{l}\text { Text } \\
\text { No-links } \\
\text { Links }\end{array}$ & $\begin{array}{l}109.10 \\
581.70 \\
703.94\end{array}$ & $\begin{array}{r}18.47 \\
502.98 \\
515.19\end{array}$ \\
\hline \multicolumn{4}{|c|}{$F(2,51)=5.553, p=.0066$} \\
\hline 5 & $\begin{array}{l}\text { Text } \\
\text { No-links } \\
\text { Links }\end{array}$ & $\begin{array}{r}52.40 \\
616.00 \\
803.59\end{array}$ & $\begin{array}{r}7.03 \\
667.49 \\
413.66\end{array}$ \\
\hline & $, 49)=6$. & $p=.0027$ & \\
\hline
\end{tabular}

number of links followed on any query was 28 . There was a statistically significant negative correlation between the average number of links a subject followed and the total number of queries completed $(r=-.74, p=.001)$. There was also a statistically significant negative correlation between the average number of links a subject followed and the total number of correct queries $(r=-.73$, $p=.001)$. The number of links followed slowed down query completion time for the subjects in the links group.

\section{Completion Time}

Comparisons were also made of the average times it took the subjects to complete the queries. For four out of five questions, the subjects in the links group took the longest amount of time to complete the query, followed by subjects in the no-links group, followed by subjects in the text group. In one of the questions (Question 3"The intergenerational mobility rate in Japan."), the links group took longer on average than the no-links group, but the text group took longest of all. Since the information requested was not readily obtainable from the table of contents in the paper text version, but required more searching of related topics in the paper text, this was understandable.

Table 4 lists the average number of queries completed by each group and Table 5 lists the means and standard deviations for completion time for each group for each question. One-way analyses of variance revealed statistically significant differences among the groups.

\section{Specifications}

Although the larger average number of specifications by subjects in the links group was not statistically signifi- 
cant, over both groups there was a significant positive correlation between the average number of specifications used for a query and the average number of seconds spent obtaining the answer to a query. Perhaps with a faster system, this relationship would not hold, but in this particular version of NetBook, there is significant overhead to forming highly specific queries. The correlation between average time per query and average number of specifications used is .84 $(p=.001)$.

As an illustration of this overhead, contrast the following two protocol excerpts for Question 5 from different subjects in the no-links group:

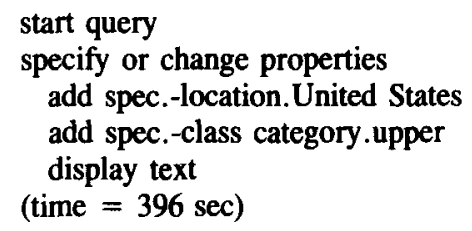

and

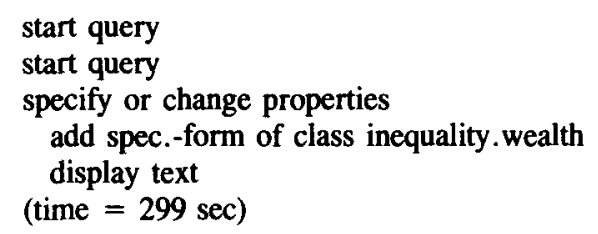

It is clear from these two excerpts from subject protocols that both subjects understood the data retrieval system. Nevertheless, neither subject used a full specification to find the answer to the question ("The percent of the national wealth that is owned by the richest fifth of Americans."'). The first subject narrowed down the range of material to be searched more, but spent so much more time doing so that retrieving the answer actually took more time (roughly one-third more).

Some subjects continued to make specifications when it was no longer necessary, or made incorrect specifications ("false starts") and then had to begin again. The following protocol excerpt illustrates the use of inappropriate continued specification. Because the user became impatient each time she asked to see text, she narrowed down the field of information requested too severely. To complete this query for Question 1 took 1,770 sec:

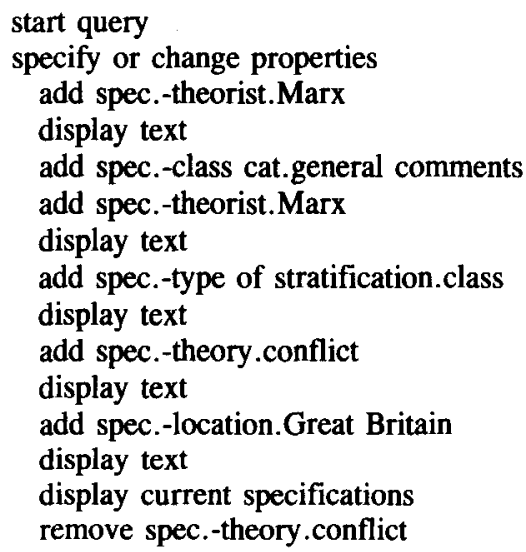

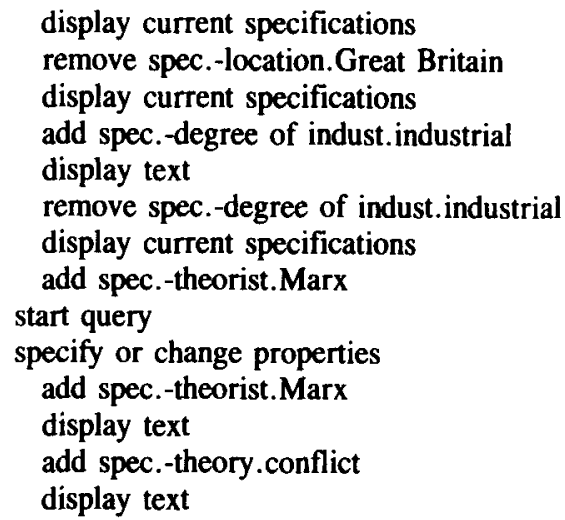

In the above excerpt, the subject decides to begin over again with a considerably scaled-down query. Her original specifications were all not entirely inappropriate and two are reused.

\section{Practice Effects}

As mentioned above, the order in which questions were presented to the subjects was rotated in all groups in order to measure practice effects. Table 6 shows mean querycompletion times for all groups combined for first through fifth queries. The subjects who began a query but did not complete it because they ran out of time are included. There appears to be a trend toward faster completion of later queries.

However, since the text group completed all queries, while only a portion of both electronic groups did so, the faster responses of the text group are overrepresented in the latter queries. Therefore, mean completion times for first through fifth queries were broken down by group and query for the subjects who had completed all five queries. The mean query-completion times are shown in Table 7. They show practice effects for both no-links and links subjects through the fourth query completed. Completion times increase for the fifth query, perhaps as a result of fatigue.

\section{Gender, Attitudinal, and Experiential Measures}

There were a total of 38 males and 22 females participating in the study. The data were broken down by gender, but there were no significant differences between males and females.

Attitudinal and experiential measures did not vary between the no-links or links groups. Eighty-eight percent of these subjects had little previous experience with databases and could thus be considered comparable on this dimension to naive or occasional users in other studies.

Table 6

Mean Query-Completion Times by Query Order

\begin{tabular}{lccc}
\hline Query Order & Number & $M$ & $S D$ \\
\hline First & 60 & 827.57 & 688.99 \\
Second & 57 & 514.05 & 483.10 \\
Third & 53 & 518.43 & 477.49 \\
Fourth & 44 & 391.18 & 438.93 \\
Fifth & 38 & 402.97 & 355.73 \\
\hline
\end{tabular}


Table 7

Mean Completion Times in Seconds by Group and Query Order for Subjects Completing Five Queries

\begin{tabular}{cccc}
\hline Query Order & Group & $M$ & \multicolumn{1}{c}{$S D$} \\
\hline First & Text & 408.90 & 576.28 \\
& No-links & 466.43 & 234.31 \\
& Links & 847.38 & 282.01 \\
& $F(2,33)=3.773, p=.0335$ & \\
Second & Text & 206.70 & 265.54 \\
& No-links & 389.83 & 166.09 \\
& Links & 543.88 & 350.13 \\
& $F(2,33)=4.334, p=.0213$ & \\
Third & Text & 257.90 & 304.03 \\
& No-links & 335.89 & 195.34 \\
& Links & 371.38 & 199.67 \\
& $F(2,33)=0.601, p=.5543$ & \\
Fourth & Text & 376.90 & 547.20 \\
& No-links & 280.33 & 88.79 \\
& Links & 300.25 & 152.93 \\
& $F(2,33)=0.338, p=.7158$ & \\
Fifth & Text & 213.60 & 219.62 \\
& No-links & 311.78 & 135.99 \\
& Links & 567.00 & 302.73 \\
& $F(2,33)=6.967, p=.0030$ & \\
\hline
\end{tabular}

Eighty percent of these subjects indicated generally favorable attitudes toward computers. There was no relationship between attitude or experience and performance.

\section{SUMMARY AND RECOMMENDATIONS}

Some questions encouraged the use of links more than others, and some subjects in the links group were more likely to use the hypertext menu than were others. This finding is consistent with that of Weyer (1982), who found that students learned simple features of Dynabook quickly, but did not use its more elaborate features.

The subjects in the no-links group completed significantly more queries than did those who used links. We believe that this is not merely a result of confusion from being offered the choice of several alternatives, because the subjects who had the option to use hypertext but did not do so performed comparably to those who did not have the option available.

Comparisons with a group that used more conventional text for knowledge exploration revealed that they performed best of all groups when the table of contents was enough to locate the desired information. When the table of contents was not enough, the text group performed poorest of the three groups. The advantage of text under certain circumstances is consistent with research reported by Shneiderman (1987). Reading from screens has been found to be roughly $30 \%$ slower than reading from paper text.

There was a statistically significant positive correlation between the average number of specifications used for a query and the average number of seconds spent obtaining the answer to a query. An excessive number of specifi- cations generally indicated an inability to properly use the system to retrieve the desired information.

There was some evidence of practice effects in the data in the two NetBook groups, through the fourth query completed. Fatigue may have increased completion time for the fifth query. There was no evidence of practice effects in the text group, but none was expected, since users of text have had plenty of prior practice and are actually expert users of the medium, rather than novices as was the case with both electronic groups.

\section{Recommendations on the Use of Links}

One of the reasons why users in a particular system have difficulty using links is that they do not always have all of the information that they need or not all of it is salient. As Landauer et al. (1982) have pointed out, users may receive only a small amount of local detail at any one time (e.g., what a fragment's links look like), whereas more global information on the overall structure of the database and its paths of links might be more useful. This is consistent with the subjects' reactions to NetBook. Some subjects expressed the fear that using links would get them too far afield. They felt it was quicker to search through the fragments called up by properties and values (which are global information) rather than to follow links through "unknown territory."

Although using links may in fact take longer and may result in a higher number of naive users getting lost, designers of information systems agree that it is highly desirable that a system have this feature, particularly when properties and values don't fit what the user has in mind. Links, however, could possibly be improved in one of the following ways:

1. A facility for allowing the user to see the properties and values of a specific link target could be helpful. With this feature, those who felt reasonably confident about their original query specifications and did not want to wander off into a different topic might not choose a particular link. On the other hand, users who felt that the properties and values of the link fragment were more appropriate than their original specifications could then modify the direction of their search. In the present study, the users of links sometimes wandered out of their specification set. They were not only uncertain about whether they had actually left their original specification set, but also uncertain about how to get back to their original specifications or even about whether it was desirable to get back to the original specification set. These difficulties could be alleviated somewhat with the proposed facility.

2. A "fisheye" view of the database, as proposed by Furnas (1983), could be presented in a small window on the user's screen. Users would have a simultaneous view of both the parts of the database structure near their current focus of interest (e.g., a fragment's links) and a view of the higher structure of the database (e.g., major attribute headings and where a fragment specification fits into that overall structure). This may be a useful way to keep 
people oriented in an information system like Netbook, whose structure is too large for naive users to deal with at one time. In the present study, some users of the links reported feeling overwhelmed by the attempt to keep themselves oriented in the text, often just giving up and randomly exploring. Orientational aids in the system would help prevent random exploring when a directed search to find specific information was more appropriate.

3. Embedding links directly into text, as Shneiderman or Brown describe (Brown, 1986a, 1986b; Shneiderman, 1987), or displaying link names at the bottom of the screen while a text fragment is displayed, might be a more efficient means for entering into a linked path than requesting a separate hypertext menu as must now be done with NetBook. Some users in the present study seemed to have difficulty moving between the specification menu and the hypertext menu. If all choices were displayed on the screen at once, it would eliminate this difficulty.

In addition, the way the data are organized in a text database may need to be examined experimentally. The categorization of attributes and values of text segments should perhaps initially be made by a group of naive users similar to the users of the system. The optimum number of properties and/or values should also be examined experimentally. In addition, the optimum amount of hierarchical structure could be explored. This may vary from application to application.

Finally, whether or not links are desirable may depend on how a system is to be used. In information systems, undirected explorations without an answer to a specific question in mind are different from directed searches for a specific piece of information. Links may be more useful when searches are undirected. A person who is generally interested in a topic might follow links that pique curiosity, without worrying too much about getting lost. But faced with a specific question and a set of properties and values to which the query can be mapped reasonably well, the user may be better off not using the links.

\section{REFERENCES}

ACKSCYN, R. M., MCCRACKEN, D. L. (1984). The ZOG approach to database management. Proceedings of the Trends and Applications Conference: Making database work (pp. 217-225). Silver Spring, MD: Computer Society Press.

AsKSCYN, R. M., McCracken, D. L., \& YoDer, E. A. (1988). KMS: A distributed hypermedia system for managing knowledge in organizations. Communications of the $A C M, 31,820-835$.

Brown, P. J. (1986a). Interactive documentation. Software-Practice \& Experience, 16, 291-299.

Brown, P. J. (1986b). A simple mechanism for authorship of dynamic documents. In J. C. van Vliet (Ed.), Text processing and document manipulation: Proceedings of the International Conference at the
University of Nottingham (pp. 34-42). Cambridge: Cambridge University Press.

BusH, V. (1945, July). As we may think. Atlantic Monthly, pp. 101-108. Canas, A. J., Safayeni, F. R., \& Conrath, D.W. (1985). A conceptual model and experiments on how people classify and retrieve documents. Paper presented at the 8th Annual International ACM SIGIR Conference, Montreal.

Cattell, R. G. G. (1980). An entity-based database user interface Proceedings of the ACM SIGMOD Conference on Management of Data (pp. 354-363). New York: Association for Computing Machinery. Conkun, J. (1987). Hypertext: An introduction and survey. IEEE Computer, 9, 17-41

Dumais, S. T., \& Landauer, T. K. (1984). Describing categories of objects for menu retrieval systems. Behavior Research Methods, Instruments, \& Computers, 16, 242-248.

ENGELBART, D. C. (1984). Collaboration support provisions in AUGMENT. In C. U. Greaser (Ed.), AFIPS Office Automation Conference Digest (pp. 51-58). Los Angeles: AFIPS Press.

FoGG, D. (1984). Lessons from a 'Living in a Database' graphical query interface. In Proceedings of the ACM SIGMOD Conference on Management of Data.

Frankhuizen, J. L., VRins, N. (1980). Human factors studies with Viewdata. Proceedings of the Ninth International Symposium on $\mathrm{Hu}$ man Factors in Telecommunications (pp. 1-7).

Furnas, G. W. (1983). Small views of large structures. Paper presented at Workshop on Intelligent User Interfaces, NH.

Herot, C. F. (1980). Spatial management of data. ACM Transactions on Database Systems, 5, 493-513.

Landauer, T. K., Dumais, S. T., Gomez, L. M., \&urnas, G. W. (1982). Human factors in data access. Bell System Technical Journal, 61, 2487-2509.

LowE, D. G. (1985). Cooperative structuring of information: The representation of reasoning and debate. Intemational Joumal of ManMachine Studies, 23, 97-111.

Motley, M. T. (1987, February). What I meant to say. Psychology Today, pp. 24-28.

Negroponte, N. (1979). Books without pages. ICC International Conference on Communications, 4, 56.1.1-56.1.8.

NELSON, T. (1984). Literary machines. Unpublished manuscript.

Shasha, D. (1985). NetBook-a data model to support knowledge exploration. In A. Pirotte \& Y. Vassiliou (Eds.), Proceedings of the 11th International Conference on Very Large Data Bases.

Shasha, D. (1986). When does non-linear text help? In L. Kirschberg (Ed.), Proceedings of the First International Conference on Expert Database Systems (pp. 109-120). University of South Carolina, Institute of Information Management, Technology \& Policy.

ShNeIDERMAN, B. (1987). User interface design and evaluation for an electronic encyclopedia. In G. Salvendy (Ed.), Second International Conference on Human-Computer Interaction (pp. 207-224). Amsterdam: North-Holland.

TriGg, R. H., \& WEISER, M. (1986). TEXTNET: A network-based approach to text handling. ACM Transactions on Office Information Systems, 4, 1-23.

WALKER, D. E. (1981). The organization and use of information: Contributions of information science, computational linguistics and artificial intelligence. Journal of the American Society for Information Science, 32, 347-363.

WEYER, S. A. (1982). The design of a dynamic book for information search. International Journal of Man-Machine Studies, 17, 87107.

YANKELOVICH, N., \& MEYrowitz, N. (1985). Reading and writing the electronic book. IEEE Computer, 18, 15-32. 